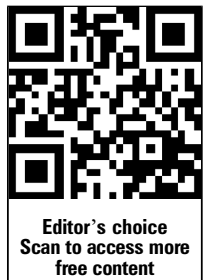

${ }^{1}$ School of Public Health, Sydney Medical School, University of Sydney, Sydney, Australia

${ }^{2}$ Bradford Institute for Health Research, Bradford Teaching Hospitals NHS Foundation

Trust, Bradford, UK

${ }^{3}$ Institute for Psychological Sciences, University of Leeds, Leeds, West Yorkshire, UK

\section{Correspondence to} Dr Reema Harrison, School of Public Health, Sydney Medical School, University of Sydney, Sydney NSW 2006, Australia; reema.harrison@ sydney.edu.au

Received 25 August 2013 Revised 27 May 2014 Accepted 28 May 2014 Published Online First 20 June 2014
CrossMark

To cite: Harrison $R$, Anderson J, Laloë P-A, et al. Postgrad Med J

2014;90:439-445.

\title{
Mentorship for newly appointed consultants: what makes it work?
}

Reema Harrison, ${ }^{1}$ John Anderson, ${ }^{2}$ Pierre-Antoine Laloë, ${ }^{2}$ Marta Santillo, ${ }^{3}$ Rebecca Lawton, ${ }^{3}$ John Wright ${ }^{2}$

\begin{abstract}
Background Mentorship has been identified as a beneficial practice for doctors and may be particularly valuable for newly appointed consultants. It is associated with a number of potential clinical and non-clinical gains, such as enhanced job satisfaction and well-being. Despite strong support, many formalised schemes fail to launch or gain momentum. Research to date has largely focused on the gains associated with mentorship but has lacked study of the factors that facilitate uptake and maintenance of

postgraduate medical education. ${ }^{3-5}$ Mentoring is championed due to the medical ethos of sharing information, experience and wisdom. ${ }^{6}$ In medicine, mentorship is often defined as:

The process whereby an experienced, highly regarded, empathic person (the mentor), guides another individual (the mentee) in the development and re-examination of their own ideas, learning, and personal and professional development.
\end{abstract} mentoring relationships by physicians.

Objectives To explore perceptions of mentorship, the extent to which UK doctors appear to value mentorship and factors that may contribute to its successful use. Design Qualitative, descriptive, multi-centre study. Sample 30 doctors including registrars, those newly appointed to consultant grade, senior doctors and medical leaders from nine hospitals in the north of England.

Method Semistructured individual interviews were undertaken between August and December 2013.

Results Findings revealed a demand for mentorship for new consultants, with widely recognised benefits associated with its use. Several factors were identified as critical to successful mentorship relationships, including consistent understanding and expectations of mentorship between mentee and mentor, positive prior experiences, a suitable match between mentee and mentor, making time for people to act as mentors and the ensuring that mentors can meet a diverse and changing set of needs.

Conclusions Mentorship for newly appointed consultants is valued, but current models of mentorship may suffer from rigid structures, mismatched expectations of participants and the absence of a culture of mentorship from training into practice. A social network approach, in which doctors have the opportunity to engage with a range of mentors through informal and naturally occurring relationships, may be one way to encourage successful and sustained mentoring relationships among doctors.

An organisational culture in which mentorship is permitted and is the norm may enable such approaches to be widely adopted.

\section{INTRODUCTION}

Mentorship occurs in different forms including among peers and within working groups, but is more commonly thought of as a one-to-one relationship. It is conceptualised in various ways depending on its context, content and purpose. ${ }^{1}$ Mentors in any capacity can generally provide psychosocial support functions (eg, counselling and role modelling) and career support functions (eg, sponsorship and coaching) to a less experienced person. ${ }^{2}$ The principle of mentoring as a learning relationship has led to its use in undergraduate and
Mentorship has been identified as beneficial for doctors internationally and across specialities. ${ }^{8-14}$ Literature reviews suggest mentoring can enhance technical and non-technical skills, which may lead to improvements in care quality and patient safety. ${ }^{8} \quad 10 \quad 15-17$ Having a mentor may encourage openness about mistakes and provide new consultants with support to avoid or report threats to patient safety. ${ }^{18-20}$ Mentees also report improved confidence, job satisfaction, working relationships, problem solving abilities, sense of collegiality and support in making career decisions. ${ }^{8}$ Gains in wellbeing are consistently cited, including reduced job stress, burnout and absenteeism. ${ }^{10} 16$ Mentors (who are often the most experienced and knowledgeable team members) may benefit from improved job satisfaction, organisational commitment, job performance, career success and reduced turnover intent. ${ }^{21}$ Mentorship may therefore contribute to creating doctors who deliver better quality and safer care.

Mentorship may be particularly valuable for doctors newly appointed to consultant grade. Recent work indicates that, in a position of newfound responsibility, newly appointed consultants may be exposed to greater job stress due to perceived deficiencies in leadership and organisational skills. ${ }^{22-24}$ The stresses associated with being a newly appointed consultants make this group more prone to error. ${ }^{25}$ In the EU, the European Working Time Directive (EWTD) was fully implemented for doctors in training in August 2009. ${ }^{26}$ The EWTD limits workers to a maximum 48-h week, averaged over a 6-month period and identifies minimum requirements in relation to working hours, rest periods and annual leave. ${ }^{27}$ Concerns have been raised that shorter working hours as a result of the EWTD may threaten patient safety. ${ }^{28}$ Doctors who are newly appointed to consultant grade have

${ }^{\mathrm{i}} \mathrm{A}$ consultant is a senior hospital-based physician or surgeon who has completed all specialist training. Consultants are responsible for and oversee the care of patients referred to them. 
reported increased fatigue, a higher number of serious medical errors and lapses in attention following the introduction of the EWTD. ${ }^{29}$ Mentorship may provide the support and guidance needed for newly appointed consultants to address these challenges.

Despite strong support for the concept of mentorship, many medical mentorship schemes historically (and currently) fail in implementation, lack visibility, or struggle to gain and maintain momentum. $^{20}{ }^{30}$ Mentorship schemes are resource intensive and require significant financial and personnel investment. ${ }^{31}$ As such, a lack of uptake or maintenance of mentoring relationships may come at great cost to healthcare organisations. This has been identified as a barrier to widespread adoption of schemes by healthcare organisations. ${ }^{31}$

\section{Conceptual framework}

There are many models of mentorship, supported by different frameworks and theoretical positions. ${ }^{32}$ In this study, we have used Garvey's conceptual framework of the dimensions by which mentorship may operate. ${ }^{33}$ This framework was selected as a useful standpoint for addressing the research objectives as it facilitated exploration of different types of mentorship relationships that may be successful or desirable for our population. Five fundamental dimensions of mentoring relationships are identified: the scope of the conversation (open/closed), who knows about the relationship and what is talked about within it (private/public), how formal and structured the conversations are (formal/informal), who takes action in the relationship (active/passive) and the predictability and reliability of the behaviours of those involved (stable/unstable). Garvey suggests that in each mentoring relationship, a unique combination of these dimensions may be present and desirable. ${ }^{32} 33$ Using these dimensions, the framework is identified as valuable for exploring the operation and conduct of mentorship schemes. It was also developed and used in a National Health Service (NHS) context.

\section{Present study}

While mentorship for newly appointed consultants may be beneficial for consultants' well-being and care quality and patient safety, few studies describe the conditions that facilitate effective and sustained engagement with mentorship for this group. Exploring our data against Garvey's framework, we aimed to understand the expectations and needs of advanced trainees, new consultant mentees and potential mentors (senior doctors). Recognising the unique and changing demands of participants may lead to more sustained mentoring relationships for doctors and address some of the challenges of mentoring in this context. ${ }^{17} 2133-35$ The research objectives were:

- To explore the extent to which UK doctors appear to value mentorship.

- To capture doctors' perceptions and expectations of mentoring (including the privacy, formality and the actions of participants).

- To identify factors that may influence doctors' decision to engage in mentorship and sustain mentorship relationships.

\section{METHODS}

\section{Setting}

The Yorkshire and Humber region in the north of England has a population of five million people and comprises 14 acute care hospitals, in addition to other community, mental health, ambulance and primary care services.

\section{Design}

A qualitative, descriptive, multi-centred interview study was conducted between August and December 2013. The sample was purposively selected to capture views of doctors who were (a) senior medical leaders or senior doctors in their department (who would be in a position to mentor new staff) and (b) new consultant appointees or registrars in nine acute hospitals. This approach was selected to provide a comprehensive account of mentorship (or its absence) for new consultant appointees as experienced and/or perceived by the participants in this sample. ${ }^{36}$

\section{Recruitment}

Invitations though email were sent to chief executives of all acute hospitals $(n=14)$ in the Yorkshire and Humber region, inviting participation. Nine hospitals agreed to participate. A further email invitation was sent out by the chief executives' offices to eligible doctors in their hospitals. Potential participants contacted the research team directly and were stratified by gender and hospital to ensure a representative sample. Only a small number of registrars were recruited because the focus of this work was on perceptions and experiences of newly appointed consultants rather than those still in training. However, we felt it valuable to capture the perspectives of a limited number of registrars who were preparing to apply for consultant posts. Data collection and analysis were an iterative process that enabled the research team to recruit until data saturation.

\section{Procedure}

Semistructured interviews allowed indepth data to be gathered with the flexibility to pursue interesting lines of enquiry. Face to face or telephone audio-taped interviews lasted between 30 and $50 \mathrm{~min}$. The interview schedule included four core topics exploring: (1) the perceived value of mentorship (eg, 'What impact do you think having a mentor might have on you, your work or how you work within your team and the organisation?), (2) interpretations and expectations of mentorship (eg, 'What kinds of things would you expect from a mentorship relationship?), (3) the factors that appeared to influence beliefs about mentorship (eg, 'What would be your main concerns about having/being a mentor as/for a newly appointed consultant (probe-why) ?) and (4) factors that appeared to influence the way that mentorship occurs (eg, 'How would you like a mentorship scheme to operate-what would be important for you?'). The schedule was reviewed and refined following pilot work with senior doctors and a pilot interview with a new consultant doctor. Participants were asked if they were aware of a mentorship scheme in their hospital and, if so, if they had been involved in this; one of two avenues of questioning then followed depending on the response. Where a mentorship scheme was operational within a hospital, participants were asked about purpose, content and delivery and about positive or negative experiences of it. Where no scheme was in place, participants were asked about the desire and perceptions of mentorship and factors that would influence their engagement in mentorship.

\section{Ethical approval}

As a needs assessment with NHS staff, NHS ethics approval was not required for the undertaking of this work. The work was conducted in accordance with ethical guidelines for educational research. ${ }^{37}$

\section{ANALYTIC STRATEGY}

Thematic analysis allows a theoretically-flexible approach for identifying, analysing and reporting patterns in qualitative data 
and is useful for obtaining detailed insights into complex issues. ${ }^{38}$ Transcripts were read by one researcher (RH) who, once familiar with the breadth and depth of content, undertook a focused line by line analysis. Commonly occurring themes that related to the study objectives and broader conceptual framework were identified. Themes were grouped under broader categories that were then labelled with reference to the conceptual framework. A subset of six transcripts was coded separately by additional researchers (PAL, JA) to validate themes and categories identified by RH. Different interpretations were resolved through discussion.

\section{RESULTS}

The sample comprised of 31 participants including: medical directors (five), deputy medical directors (four), clinical directors (six), recently appointed consultants (13), and registrars (three). Six themes emerged: (i) Mentorship as protective, (ii) Variability in the perceived role and function of mentorship, (iii) Informal and multiple mentors, (iv) The importance of 'personalities', (v) Mentoring as part of professional identity and (vi) Permission to mentor.

\section{Mentorship as protective}

Respondents indicated a need and desire for mentoring new consultant appointees. Of all the purposes of mentoring noted, the most critical for medical leaders was to mitigate patient harm. Mentors who offered guidance and support were recognised as a safety net that might reduce the likelihood of errors, and, furthermore, a vehicle for supporting recently appointed consultants who are involved in an adverse event.

\footnotetext{
I think mentorship is inevitably linked to quality and safety because people need to have someone to go to, a sounding board, someone to ask when they think they can't go to anyone or if they have to deal with complaints. (Medical leader 8)

We anticipate that a mentor scheme is a protective scheme for the new consultant and for the patients receiving the treatment. So if you've got a healthy engaged consultant...then you're likely to have a safer doctor. (Medical leader 6)
}

Registrar and new consultant appointees discussed heightened job stress that resulted from being in a newfound position of responsibility. Mentorship was valued as a strategy to manage the emotional burden of their role.

\begin{abstract}
If you are a new consultant and you'll be faced with challenges... you've not come across before... the management, leadership side of things ...you're allegedly where the buck stops...I think [a mentor] would certainly help. (Registrar 2)

It's the non-clinical side...managing your time...managing colleagues and tricky situations... that it would be good to...that you usually need advice on. (New consultant 4 )
\end{abstract}

\section{Variability in the perceived role and function of mentorship} Respondents typically understood mentorship to mean the provision of support and guidance from a more senior and experienced clinician to a newer consultant. Mentorship was generally conceptualised as a one-way relationship, with the mentee the main benefactor; respondents rarely considered that mentors could benefit from the relationship.

Having somebody that can offer you guidance and support and look up to, who is further in their career than you are. (New consultant 1)
To be a mentor you probably have to be in post yourself for five years or more to be able to provide appropriate support. (Medical leader 1)

While there was consensus around the broad goal of mentorship, expectations of the mentoring relationship varied. Respondents recognised the lack of consistent understanding of the term among doctors and described confusion around distinguishing mentorship from other relationships such as coaching and peer support.

Well what's mentoring what's coaching what's peer support... what is a mentor? (Medical leader 2)

All new consultants are meant to be appointed a mentor-is it a mentor or a coach actually? (New consultant 2)

Medical leaders generally described mentorship as a forum for non-clinical personal development and for discussing professional issues, such as learning how to manage workload, leadership and managerial roles.

The clinical guidance... was more guidance of how to handle difficult clinical situations which have nothing to do with the clinical management of the patient (Medical leader 3)

It's sort of the non-clinical side of things I think could be really useful to either have a mentor. (Senior doctor 3)

Surgical senior doctors uniquely perceived mentorship as a resource for guiding clinical skills development and saw mentors as a point of contact to discuss how to approach difficult or new clinical cases; this is understandable as mentorship in surgical disciplines is common.

They may become a new consultant and they may have very little experience of doing a hysterectomy.... and actually need a consultant colleague to actually assist them in doing that operation themselves. (Medical leader 2)

Because of the way that the training has been abbreviated and streamlined quite a few of junior consultants...have insecurities about their practice and still would like to have somebody more senior...in a surgical specialty to assist them in theatre. (Medical leader 5)

Registrars and recently appointed consultants described a wide range of beliefs about the purpose of mentorship. Respondents identified support with networking, organisational sign-posting, support with personal and inter-personal relationships (including professional relationships), insight into the personalities and behaviours of existing team members and guidance around clinical skills and knowledge.

Somebody...that can talk to you about how to get through and the people to know .... and therefore you can network a little bit more. (New consultant 1 )

I don't think it's the clinical stuff, it's more the non-clinical stuff. (New consultant 3)

Critical for the sustainability of the mentoring relationship was the extent to which both shared a common understanding of the goal of mentoring; mismatched expectations were identified as problematic.

A lot of these schemes probably don't work because people don't realise what mentorship is... or what their role was supposed to be. (New consultant 4 )

You'd need to have a clear description... so what would be the expectations of the mentor... and then in return for that, 
what might the mentee be doing for the organisation. (Medical leader 1)

\section{Informal and multiple mentors}

Current or past experiences of influential relationships with senior clinicians were often described-akin to the characteristics of mentorship. It became apparent that while mentorship schemes are not common (only two organisations had a scheme), most clinicians could recall exposure to some form of mentoring during their training or practice. Recently appointed consultants reported engaging with a variety of colleagues at the start of their consultant post, often identifying those who could help with personal and broader professional issues and others who could guide them in the development and use of clinical skills and knowledge. Medical leaders also recognised that more than one mentor might contribute to a newly appointed consultant's development. The opportunity to have multiple mentors, each serving a different purpose, emphasised a wide range of issues confronting recent consultant appointees as well as registrars, who as a group described a diverse and evolving set of mentee needs. These comments reiterated the importance of mentorship being nuanced and flexible.

In my last job...I had about four or five different mentors that probably didn't even realise they were to me ...I chatted to different people about different things in order to get the guidance that I needed. (New consultant 4)

I draw on different people for different bits of information and I think a lot of my colleagues do. (New consultant 5)

I think in an ideal world it would be two mentors, you'd have a clinical mentor and a non-clinical mentor. (Medical leader 6)

Multiple mentors were recognised as valuable, but this was qualified by the insufficient numbers of senior staff available to take on mentoring roles and the difficulty of matching mentee and mentor. In particular, senior consultants who engage in a range of non-clinical activities are likely to be those in high demand from mentees and already overstretched.

...what prevents these things from happening is how you'd find the appropriate people to be the mentors and then how you would resource that...I think the thing that stopped us doing it has been effectively time and cost. (Medical leader 1)

\section{Mentoring as part of a professional identity}

Experiences as a trainee and socialisation in the medical profession were important. New consultants in specialties such as anaesthesia and gastroenterology described how experiences during training socialised them into operating via a mentoring model; turning to senior team members for support and guidance became a part of their professional identity.

Anaesthetics is quite a formalised training scheme ... throughout that time there's always been people, I suppose you'd call them mentors or call them role models, who are people who are always accessible for contact. (New consultant 6)

It's a really tight gastro department...I would consider a lot of what I get is mentorship... everybody seems to have a little to offer. (New consultant 7)

\section{Permission to mentor}

The acceptability of mentoring in teams and specialities appeared to be influenced by the structure and culture of the working environment. In some smaller departments, where teams were close-knit, informal mentoring occurred within a supportive team environment. In such cases, mentoring was readily available for recent appointments joining the team.

We're very lucky with our department...I've worked in other departments where it...wouldn't be thought of as quite so reasonable to go and speak to a colleague and say I've got this tricky case, what do you think about that? (New consultant 3)

Because it's a relatively small unit, it's quite friendly and I have quite a lot of interaction with one of the other consultants in the same specialty as me so in a way he's kind of an unofficial mentor, I go and ask him about things. (New consultant 9)

Alternatively, where mentoring did not occur organically, mentorship schemes were imposed using a more formalised structure. A formal approach to mentoring created challenges including ensuring the compatibility of mentee and mentor, assigning a mentor who could meet the mentee's expectations and having inadequate knowledge of one another before commencing the mentoring relationship.

I don't [have a mentor] and I should have done....It never quite happened when I started and I was given a name and contact details for someone about six to eight months into the job by which time I was just so busy I never had a chance to follow it up. (New consultant 2)

We had a buddy scheme...they linked you up with a person ... who was supposed to help you ....it often depended on how well you got on with them. I met up with my buddy twice and then that was the last I saw of them. (New consultant 4)

The need for significant organisational support when establishing a formalised mentorship was recognised, including the need for organisational buy-in to build a culture of mentorship.

The majority of the challenge would be time and cost, how you'd persuade people to effectively give up doing something else to do it. (Medical leader 1)

Who is going to organise it, who's going to allocate mentors, who's going to ask for volunteers to be mentors and then teaming people up. There is a big drain on people's time and lots of demands.... (New consultant 8)

\section{The importance of 'personalities'}

A number of practical issues were reported as influential in respondents' desire to engage in mentorship. The degree of formality and privacy in the relationship were frequently discussed, but respondents indicated that the personalities of the mentor and mentee and the degree to which each one complimented the other was the most important. Compatibility was described as critical to establishing and maintaining a mentoring relationship. Most respondents identified a need for 'character' matching.

For me the most successful mentoring relationships have been the ones where I've got on well with that person and I think that's where it fails, if you don't. They have to be somebody that's got an awful lot in common with you. (New consultant 4)

I've found that it's really how you get on with someone in the relationship... and that's not something you can pick out from a list... nor can that be someone is allocated to you. (Registrar 2)

This need for compatibility raised challenges. The difficulty associated with an allocated mentorship in the context of coupling compatible personalities was widely acknowledged. Respondents commonly suggested that mentees have access to an available cohort or pool from which they could identify a 
possible mentor as one way for them to maintain some degree of choice when embarking on the relationship.

...it would be a well-publicised list of people that you could speak to and get in contact with and see how you get on and take it from there. (New consultant 3)

If there were a list of people that were happy to offer advice... and then you could approach somebody yourself. (New consultant 4)

\section{DISCUSSION}

The benefits of mentoring doctors are widely documented. ${ }^{20} 39$ It is considered a core aspect of continuing professional development, but many schemes lose momentum or fail to begin. $^{6} 11 \quad 1720 \quad 40$ Through interviews with UK doctors, we aimed to explore the extent to which our sample values mentorship, capture doctors' perceptions and expectations of mentoring and identify factors that may influence their decision to engage in and sustain mentorship relationships. A range of factors appeared to be important in determining successful and sustained mentorship. Findings are summarised in relation to the research objectives, with implications for research and practice.

Towards objective one, mentorship was identified as a valuable resource for supporting recently appointed consultants' clinical and non-clinical skills development, for managing heavy workloads and professional relationships. ${ }^{16}$ If effectively implemented by healthcare organisations, mentors may provide guidance and support for new consultants that enhance patient safety. ${ }^{41}$ With regard to objective two, expectations of the formality, privacy and scope of mentorship varied within our sample. Potential mentees indicated preference for less formality, more privacy and a broader scope. Agreeing the terms of the mentorship along these dimensions at the outset may be critical to a successful and sustainable relationship. ${ }^{33}$ Towards objective three, past experiences of mentoring, professional socialisation, the opportunity to engage with multiple mentors and positive appraisals of mentorship were influential in doctors' decision to engage in and sustain mentorship. Suitability of the match between mentee and mentor, the acceptability of mentorship in the working environment and the career stage at which mentoring began were also important for both parties, reflecting the broader literature. ${ }^{164344}$

\section{Implications}

Our findings are important in the context of existing work that has explored the value of mentorship during clinical training and for enhancing patient safety. However, as illuminated in the context of clinical supervision, these gains are reliant on senior clinicians having the necessary patient safety knowledge themselves. ${ }^{45}$ Difficulty distinguishing mentoring from other developmental activities (eg, supervision and coaching) was evident, echoing existing literature. ${ }^{8} 46$ Mentorship and supervision were often used interchangeably by participants. Challenges of conflicting expectations of mentorship may be minimised by a clearer understanding of the distinction between the role of a clinical supervisor to guide, assess and enhance clinical practice and the role of a mentor which may be much broader and not tied to clinical assessment.

In the context of Garvey's model, our findings contribute knowledge of the factors that may lead to successful and sustained mentoring relationships for newly appointed consultants. This knowledge is critical to inform programmes that use mentoring as part of continuing professional development.
Compatible expectations regarding formality, scope and privacy of mentorship are essential and may be best achieved where there is an opportunity to create a unique mentorship relationship that fits the needs of both parties. New appointees and registrars indicated a preference for not just one mentor but several mentors to meet their diverse range of needs. Medical leaders often use allocation to ensure even distribution of workload among senior staff, but the allocation may create challenges for finding compatible mentors and mentees. Allocating a mentor and mentee may limit the extent to which the formality, scope and privacy of the relationship can be mutually agreed to suit the participants, resulting in incompatible and unsustainable relationships. Such an approach may limit the uptake and sustainment of mentoring relationships and must be adopted with care. $^{33}$

Garvey identifies mentees' needs and expectations as broad and varied; it would be difficult for a single mentor to meet this range of needs alone. Loosely formalised relationships with supportive senior staff during education and early career (in the context of a supportive team environment) may contribute to an evolved culture of mentorship among new consultants. A mentorship culture is likely to develop when senior colleagues and peers informally create a culture of collegiality, in which informal advice and support can be sought. This social network approach to mentoring may more adequately meet each new appointee's unique and changing set of needs and at the same time distributes the mentoring workload evenly among staff. Peer support has also been identified as an acceptable and useful strategy and a valuable component of a supportive infrastructure. $^{47} 48$

Informal mentoring requires an organisation that encourages and permits time to be spent on mentorship activities but requires no formal funding and or job-plan incorporation. The extent to which informal approaches to mentoring are currently used is difficult to quantify, but an organisational culture in which mentorship is permitted (by allowing staff sufficient time and resource) and is the norm may enable this approach to be widely adopted. One mechanism for achieving this in the NHS may be through building mentoring in as an expectation for annual appraisal. This field of study may benefit from further work that focuses on the circumstances and organisational environment which give rise to mentoring that arises organically (and operates informally). Future work may also draw comparisons between practitioners' perceptions that have or have not experienced mentorship to determine the impact of being mentored on beliefs and expectations.

\section{Limitations}

The interviews were undertaken in the NHS in one region of England, limiting the extent that our findings can be generalised. Many respondents were recently appointed or senior staff, which may have inhibited or modified responses. Some hospitals in the study region had existing schemes, which may have distorted perceptions of their value in either a positive or negative way depending on experiences. Interviews were conducted by three of the authors (RH, PAL, JA); one was a university researcher with a background in Psychology and two were registrars undertaking a year out of their clinical roles to train in quality and patient safety research at a health research institute. Participants' contributions may have been shaped by their perceptions of the interviewers. Participants may have been more or less open and frank depending on their perceptions of the interviewers' roles and their relationship to the health service. There was potential for the researchers to also shape the data by 
their own beliefs and views on the topic. We minimised the potential for researcher bias by ensuring researchers did not contribute their thoughts or beliefs before, during or after data collection. Participants were fully informed that the researchers were independent from their health service roles in this study, of their anonymity as participants and that the study was not being undertaken for a particular NHS organisation.

\section{CONCLUSIONS}

Mentoring for new consultants is valued, yet many current models suffer from rigid structures, mismatched expectations of participants and the absence of positive mentoring experiences in training. Providing mentees with an opportunity to engage with a range of mentors in supportive work environments may be the best springboard for establishing and sustaining mentoring relationships for new consultants as part of continuing professional development.

\section{Main messages}

- Mentorship may be beneficial for newly appointed consultants who may be vulnerable to stress and error.

- Mentorship for doctors that supports the development of technical and non-technical skills may lead to improvements in care quality and patient safety.

- Doctors may be encouraged to engage in mentorship if the level of formality, privacy and scope of mentorship are matched to mentor and mentee expectations and needs.

- Early introduction to mentorship during training and an informal network of mentors within the workplace may be useful ways to encourage newly appointed consultants to access and maintain mentorship relationships.

\section{Current research questions}

- How can healthcare organisations encourage the use of informal mentorship?

- What are the challenges of informal mentorship?

- Is there a measureable improvement in patient safety outcomes as a result of mentorship?

\section{Key references}

- Oxley J. Mentoring for physicians: a look at the literature. Physicians' Forum, 2003. http://www.ncl.ac.uk/medev/assets/ documents/Mentoringbenefits.pdf

- Sambunjak D, Straus SE, Marusic A. Mentoring in academic medicine: a systematic review. JAMA 2006;296:1103-15.

- Brown JM, Ryland I, Shaw NJ, et al. Working as a newly appointed consultant: a study into the transition from specialist registrar. Br J Hosp Med 2009;70:410-14.

- Taherian K, Shekarchian M. Mentoring for doctors. Do its benefits outweigh its disadvantages? Med Teach 2008;30: e95-9.

- Garvey B. A dose of mentoring. Educ Train 1994;36:18-26.
Acknowledgements The authors would like to thank Professor Merrilyn Walton from the School of Public Health, University of Sydney, for her contribution in reviewing this manuscript. We also thank the participants from each of the nine hospitals and the healthcare managers that facilitated this work.

Contributors Each of the listed authors made substantial contributions to conception and design, acquisition of data, or analysis and interpretation of data; drafting the article or revising it critically for important intellectual content; and to the final approval of the version to be published as set out in the Uniform Requirements for Manuscripts Submitted to Biomedical Journals. RH, RL, JW conceived and design the research idea. RH, PAL and JA were responsible for the acquisition of the data. RH, P-AL, JA and MS were responsible for the analysis and interpretation of the data. All authors contributed to the drafting the article or revising it critically and gave final approval for the version submitted.

Funding This work was an unfunded study that received institutional support from the Bradford Institute for Health Research, Bradford, UK.

\section{Competing interests None.}

Ethics approval This was undertaken as part of a needs assessment with healthcare staff in the Yorkshire and Humber region and did not require NHS ethics approval. However, the work was undertaken in accordance with ethical requirements for educational research (BERA, 2011) as stated in the manuscript.

Provenance and peer review Not commissioned; externally peer reviewed.

\section{REFERENCES}

1 Haggard D, Dougherty T, Turban D, et al. Who is a mentor? A review of evolving definitions and implications for research. J Manag 2011;37:280-304.

2 Kram KE. Mentoring at Work: developmental relationships in organizational life. Glenview, IL: Pearson Scott Foresman, 1985:1-268.

3 Buddeberg-Fischer B, Herta KD. Formal mentoring programmes for medical students and doctors - a review of the Medline literature. Med Teach 2006;28:248-57.

4 Connor MP, Bynoe AG, Redfern N, et al. Developing senior doctors as mentors: a form of continuing professional development. Report of an initiative to develop a net-work of senior doctors as mentors: 1994-99. Med Educ 2000;34:747-53

5 Frei E, Stamm M, Buddeberg-Fischer B. Mentoring programs for medical students -a review of the PubMed literature 2000-2008. BMC Med Educ 2010;10:32-46

6 Connor M, Pokora J. Coaching and mentoring at work: developing effective practice. London: McGraw-Hill, 2007.

7 SCOPME: Standing Committee on Postgraduate Medical and Dental Education. Supporting doctors and Dentists at work: an enquiry into mentoring. London: SCOPME, 1998

8 Department of Health. Mentoring for physicians. London, 2004

9 British Medical Association. Refugee physicians. London: BMA briefing note, 2006. http://www.lbma.org.uk/ap.nsf/Content/ hub+refugee+physicians

10 British Medical Association. Consultant mentoring schemes. London: BMA, 2009.

11 Humphrey HJ. Mentoring in academic medicine (Vol. 5). Philadelphia: ACP Press, 2010.

12 Steven A, Oxley J, Fleming WG. Mentoring for NHS doctors: perceived benefits across the personal-professional interface. J $R$ Soc Med 2008;101:552-7.

13 Stamm M, Buddeberg-Fischer B. The impact of mentoring during postgraduate training on doctors' career success. Med Educ 2011;45:488-96.

14 Allen TD, Poteet ML, Eby LT, et al. Career benefits associated with mentoring for protégés: a meta-analysis. J App/ Psychol 2004;89:127-36.

15 Pawson R. Mentoring relationships: an explanatory review. ESRC UK Centre for Evidence Based Policy and Practice: Working Paper 21. 2004.

16 Oxley J. Mentoring for physicians: a look at the literature. Physicians' Forum, 2003. http://www.ncl.ac.uk/medev/assets/documents/Mentoringbenefits.pdf

17 Sambunjak D, Straus SE, Marusic A. Mentoring in academic medicine: a systematic review. JAMA 2006;296:1103-15.

18 Roberts G, Moore B, Coles C. Mentoring for newly appointed consultant psychiatrists. Psychol Bull 2002;26:106-9.

19 Harrison R, McClean S, Lawton R, et al. Mentorship for newly appointed consultants: a strategy for enhancing patient safety? J Patient Saf 2013. Published Online First: 27 Feb 2014. doi:10.1097/PTS.0b013e31829e4107e

20 Ehrich L, Hansford B, Tennant L. Formal mentoring programmes in education and other professions: a review of the literature. Educ Adm Q 2004:40:518-40.

21 Ghosh R, Reio TG. Career benefits associated with mentoring for mentors: a meta-analysis. J Voc Behav 2013;83:106-16.

22 Beckett M, Hulbert D, Brown R. The new consultant survey 2005. Emerg Med Jr 2006;23:61-3.

23 Brown JM, Ryland I, Shaw NJ, et al. Working as a newly appointed consultant: a study into the transition from specialist registrar. Br J Hosp Med 2009:70:410-14. 
24 Brown JM, Shaw NJ, Graham DR. The first five years: a mixed methods study investigating reflections on working as a hospital consultant. JRSM short reports 2013;4:2042533313476686.

25 Westerman M, Teunissen PW, Fokkema JP, et al. The transition to hospital consultant and the influence of preparedness, social support, and perception: a structural equation modelling approach. Med Teach 2013;35:320-7.

26 British Medical Association. 2014. http://bma.org.uk/practical-support-at-work/ewtd [Retrieved 01 April 2014]

27 NHS Employers. 2014. http://www.nhsemployers.org/PlanningYourWorkforce/ MedicalWorkforce/EWTD/Pages/EWTD.aspx

28 Clarke RT, Pitcher A, Lambert TW, et al. UK doctors' views on the implementation of the European Working Time Directive as applied to medical practice: a qualitative analysis. BMJ Open 2014;4:e004390.

29 Murray $A$, Pounder $\mathrm{R}$, Mather $\mathrm{H}$, et al. Junior doctors' shifts and sleep deprivation: The European working time directive may put doctors' and patients' lives at risk. BMJ 2005;330:1404.

30 Hutton-Taylor S. Cultivating a coaching culture. BMJ 1999;318:S2-7188.

31 Taherian K, Shekarchian M. Mentoring for doctors. Do its benefits outweigh its disadvantages? Med Teach 2008;30:e95-9.

32 Ehrich C, Hansford B, Tennet L. Closing the divide: Mentoring theory and practice. ANZAM 2001 Conference, 'Closing the Divide; Auckland, New Zealand, 5-7 December 2001.

33 Garvey B. A dose of mentoring. Educ Train 1994;36:18-26.

34 MacLeod S. The challenge of providing mentorship in primary care. Postgrad Med J 2007:83:317-9.

35 Pololi L, Knight S, Dennis K, et al. Helping medical school faculty realize their dreams: an innovative, collaborative mentoring program. Acad Med 2002;77:377-84.
36 Sandelowski M. Focus on research methods-whatever happened to qualitative description? Res Nurs Health 2000;23:334-40.

37 British Educational Research Association. Ethical guidelines for educational research. London: BERA, 2011.

38 Braun V, Clarke V. Using thematic analysis in psychology. Qual Res Psychol 2006;3:77-101.

39 Paice $E$, Hard S, Moss F. How important are role models in making good physicians? BMJ 2002;325:707-10.

40 General Medical Council. Good medical practice. GMC Publications, 2013.

41 Sexton JB, Thomas EJ, Helmreich RL. Error, stress and teamwork in medicine and aviation: cross sectional surveys. BMJ 2000;320:745-9.

42 Garvey B. Healthy signs for mentoring. Educ Train 1995;37:12-19.

43 Jackson VA, Palepu A, Szalacha L, et al. Having the right chemistry: a qualitative study of mentoring in academic medicine. Acad Med 2003;78:328-34

44 Rose GL, Rukstalis MR, Schuckit MA. Informal mentoring between faculty and medical students. Acad Med 2005;80:344-8.

45 Walton M, Barraclough B. Clinical supervisors - are they the key to making care safer? BMJ Qual Saf 2013:22:609-12.

46 D'Abate CP, Eddy ER, Tannenbaum SI. What's in a name? A literature-based approach to understanding mentoring, coaching, and other constructs that describe developmental interactions. Human Res Dev Rev 2003;2:360-84.

47 Hu Y, Fix M, Hevelone ND, et al. Physicians needs in coping with emotional stressors: the case for peer support. Arch Surg 2012;147:212-17.

48 Scott SD, Hirschinger LE, Cox KR, et al. The natural history of recovery for the healthcare provider 'second victim' after adverse patient events. Qual Saf Health Care 2009;18:325-30 